\title{
A Study of Agile Methodology - Scrum for Cockpit Simulation
}

\author{
Zheng Liu*, Fei Li, Chunze Wu, Hongtao Liu and Jingjin Zhang \\ Shanghai Aircraft Design and Research Institute, Cockpit Integration and Industrial Design Research Department, Shanghai \\ 201210, China \\ ${ }^{*}$ Corresponding author
}

\begin{abstract}
A project management method for the simulation work has been introduced, including agile methodology, scrum framework, and cockpit simulation projects. Agile development takes the evolution of user's needs as the core, and adopts step-by-step to develop software iteratively. In the agile development, the software project is cut into multiple sub items at the beginning of the construction, and the results of each subproject are tested, with the features of visual, integrated and operational use. This design methodology has been applied in the prototype of the civil aircraft cockpit simulation.
\end{abstract}

Keywords—agile methodology; project management; cockpit simulation

\section{INTRODUCTION}

The Computer Aided Software Engineering (CASE) in the flight simulation provides the technical support for the simulation design of the aircraft cockpit widely [1,2]. Computer technology aids engineers to design the control panel, the information layout on the display screen, the alarm message inside the cockpit and the external terrain and climate environment outside the cockpit. With the simulated flight attitude or the virtual systems working modes, the pilot can accomplish various flight tasks, such as take-off, approaching, cruising, tower communication, and so on [2].

However, the cockpit simulation work integrates into many different aircraft subsystems together, such as, hydraulic system, fuel system, communication system, electricity power system and anti-ice system, which might be involved in many development groups [3]. The traditional development process like waterfall model, spiral model, and incremental model cannot satisfy the aircraft-level complex cross-functional requirement, which emphasizes more on the task division and priority of the task sequence instead of team member's collaboration and cooperation [3]. In addition, each subsystem's evaluation gives rise to the determinative changes on the cockpit design. The collaboration work and the unclear and changing requirement on the product make it much more difficult to reduce the market risk and satisfy the current fierce market competition [4].

This article introduces a kind of highly efficient and convenient project management methodology, which aids to master the inter-disciplinary or multi-functional system project.

\section{AgILe Methodology - SCRUM}

With the rapid development of technology and the globalization, the new features of software development include fast pace of development, frequent change of demand and technology and high productivity [5]. The agile methodology (also known as the lightweight method) is a general term for a set of development methods, which is characterized by its highly valued software productivity, and is mainly applicable to the development of small project groups with vague or rapid changes [6]. Takeuchi Hirotaka and Nonaka Fujiro first mentioned scrum concept, as a kind of agile development frameworks, for the product development in their article. They point out that the traditional "relay" development model has not met the fast and flexible market demand, while Scrum is a framework for developing and maintaining complex products with the incremental, iterative development process [7].

In the scrum framework, the entire development process consists of several short iterative cycles. For each iterative cycle called a Sprint, the recommended length is about 2 to 4 weeks. In the scrum working process, the product owner collects the needs, expectations, and demands from all parties to the product backlog list. Product backlog is used to collect the product to-do items, manage the requirements of the product, and priorities the development list [8]. 

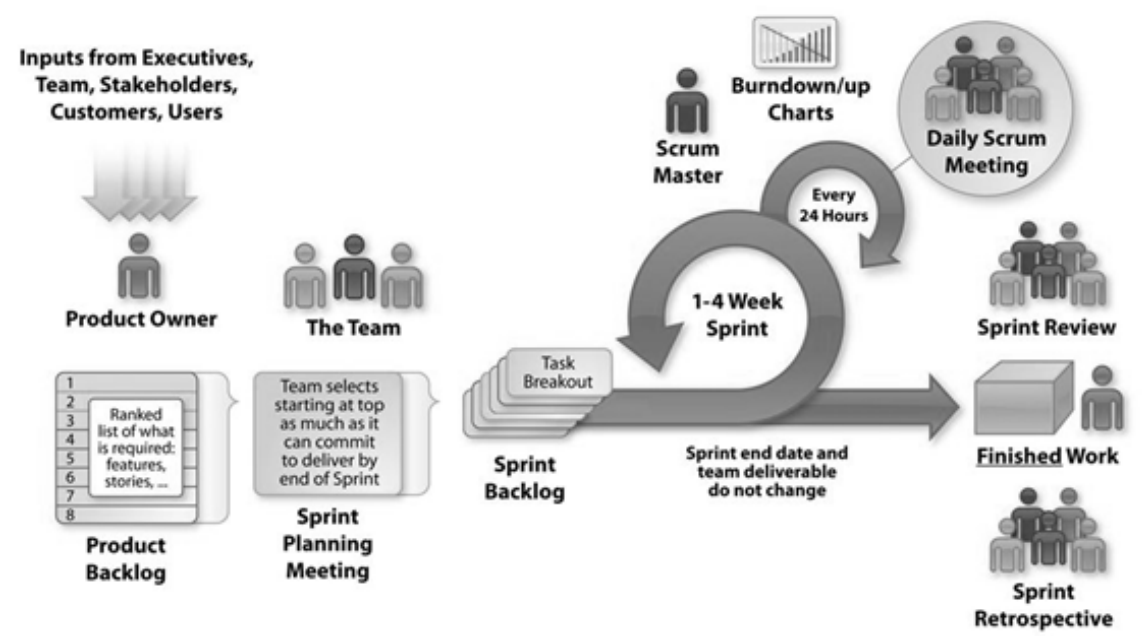

FIGURE I. THE BASIC SCRUM FRAMEWORK

At the beginning of the sprint, product owner will work with the team to select the task from the product backlog, clarify the questions raised by the team, and decompose it into sub-asks [9]. After the sprint planning meeting, the sprint backlog will be formed. For the functions on the development list of single iteration, Scrum master selects each sprint based on the team's ability and the priority in the product to-do list. A team can focus on what is going on every step of the way without interruption [9]. For a fixed time period, the team should deliver the software product to customers as far as possible during this cycle. The burn down/up diagram shows the remaining working time and the completion of the task of single iteration. To strengthen the team communication, each day team should choose a time to exchange each other's progress together, solve the problems in time and let the team at any time to understand how far we are from the sprint target [9].

At the end of the sprint cycle, a review meeting is required to enable the team to show the completed functions to the product leader and stakeholders [10]. The practice of sprint audit by team members is to display functions, answer stakeholders' questions, and notice expected changes. The sprint retrospective, usually after the review meeting, summarizes the last iteration after the end of the sprint, and puts forward some improvements, which is a continuous improvement process [9].

The basic roles in the scrum process can be classified as product owner, scrum master, and scrum member. The responsability of the role is illustrated as follows [10]:

- Product Owner: the customer in the scrum framework provides his/her requirement to the product owner. As the voice of the customer, the product owner clearly knows the vision of the product sorts out and prioritizes the to-do list and defines the acceptance criteria. After the cycle of sprint, he delivers business value to the customer through incremental product release.

- Scrum Master: the scrum master acts as the coach or the team leader, to ensure the team's reasonable operation of Scrum, and help the team to remove obstacles in the implementation.
- Scrum Team: It can be regarded as a development team and a cross functional team, who can deliver an end-to-end product that, is truly valuable to customers.

\section{CASE Study: A Project Of AircRAft COCKPIT HMI SIMULATION}

Aircraft cockpit is the primary place for the crew members to get enough information about the aircraft and the surrounding flight environment, manipulate the aircraft for each flight cycle, and guarantee the safety for each flight cycle $[11,12]$. Therefore, the rational design of the cockpit has played a key role in completing flight tasks, ensuring flight safety and providing information services. The cockpit layout of modern civil aircraft is based on the traditional "screen" and "switch", such as displays, keyboards, and buttons and so on, to meet more automatic functions of the aircraft [12]. The complexity of cockpit design has also brought more challenges to aircraft designers with the increased automatic functionality in the cockpit. In addition, how to balance the airworthiness regulations and market requirements is also a problem that designers need to consider. Only to listen to the opinions of all parties, to analyze the needs of users with different backgrounds and to optimize the operation program repeatedly, can we deliver a satisfied product [12].

The aim of the project "Aircraft Cockpit Human Machine Interaction (HMI) Simulation" is to deliver a computer-based platform to realize the rational design of the cockpit and assess or optimize the irrational aspect during the whole development process. The "screen" and "switch", such as displays, keyboards, and buttons are simulated as objects in the software application, which can be operated with the mouse or accessed by the touch screen. The operation program for the crew member is programmed with the software code or diagram, which can be easily edited and complied. The advantages are also flexible design and simple modification, but cost free.

\section{SCRUM APPLICATION}

The software project “Aircraft Cockpit HMI Simulation” has the features of flexible modification, portability, cost saving, and 
quick access to the user's requirements, which can be adaptive to the scrum framework, see figure 2 .

The input documentation or design requirement documentation includes Flight Crew Operation Manual (FCOM), HMI description for the aircraft systems in the cockpit, and related CAD documentations with basic size and shape information and so on. The whole project can be identified as the following processes: requirement check out, concept design, control panel and display development, HMI logic sort and development, engine indication and crew alarming system display (EICAS) development, and integrated testing phase.

The daily development targets are listed on the backlog according to the status in the last sprint and how does the whole project process. The scrum development team can be divided into four cross-functional groups with respective responsibilities, namely cockpit control panel \& display group, HMI logic group, aircraft model building group, and hardware architecture group. In order to increase the development efficiency and benefit the maintenance purpose, the front user interface (GUI) and background control logic (HMI logic) in this project are decoupled and developed each other separately. For each sprint cycle, the daily scrum meeting notices the problem met during the development, required documentation from the scrum and some engineering change management (ECM) communication issues.

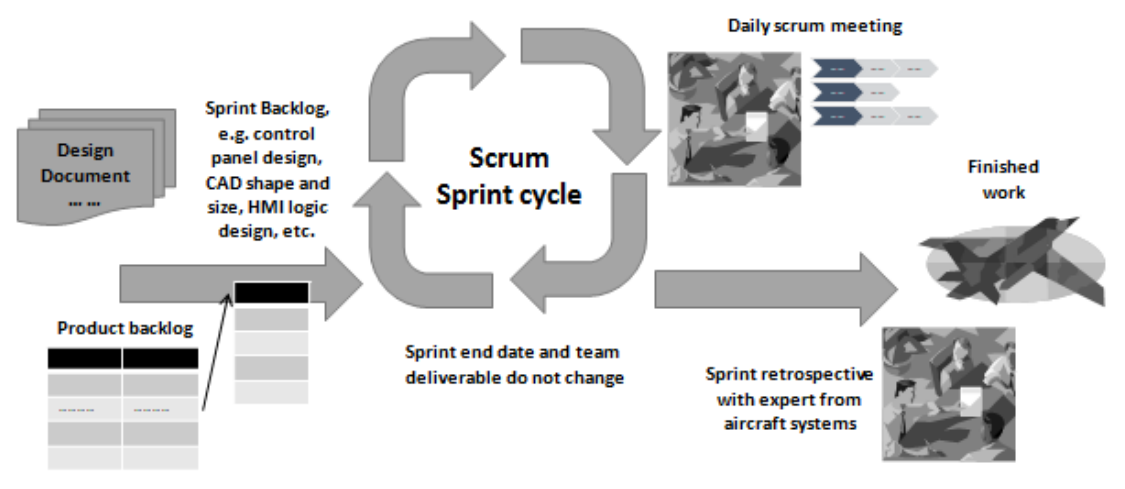

FIGURE II. THE SCRUM APPLICATION - AIRCRAFT COCKPIT HMI SIMULATION

After one completed sprint cycle, for example, the expert from the aircraft systems, such as recording and indicating system, fuel system, hydraulic system, and electricity system and so on review and evaluate the HMI logic and then feedback the comments to the scrum development team again.

\section{SUMMARY}

With the basic idea of agile design, this paper introduces a project management method - scrum, which is suitable and applicable for the aircraft cockpit simulation project.

- The focus of this lightweight design method emphasis on the engineer instead of the project or task itself [13]. Through the scrum interactive process all the team members involve in project development. The review or the retrospective phase guarantees the quality and the traceability of the whole development process.

- The other advantage of scrum is flexible design and modification [14]. Because all the development team members take part in the development phases, the issues during the whole process can be discussed and fixed for each sprint cycle.

\section{REFERENCES}

[1] Duan Lin. The evolution and development trendency of human-machine interface in cockpit [J]. Civil Aircraft Design and Research, 2017 (1): 7-11.

[2] Dong Dayong, Yu Jinhai, Li Baofeng, Chen Yingchun et al. Cockpit human factors of airworthiness verification technology [J]. Chinese Journal of Aeronautics, 2016, 37 (1): 310-316.
[3] Qiu Weilong, Chen Guoxing. Key technology for modeling and simulation of aircraft virtual instrument [J]. Aircraft Design, 2014 (3): 49-54.

[4] Jin Zhefeng, Zhang Yinbo, Liu Haiyan. Design and research of civil aircraft cabin layout design method of civil aircraft [J]. Civil Aircraft Design and Research, 2017 (1): 12-16.

[5] Hironori Washizaki, Shota Suzuki, Ryushi Shiohama. Systematic Mapping of Workshops for Learning Agile Software Development Principles, Education and Educational Research, EER 2013.

[6] Bhavya N Javagal, Ratnakala, Rajeshwari. S. N. and Nandita M. Software Development Using Agile \& Scrum [J], International Conference on Advances in Computer and Electrical Engineering (ICACEE’2012) Nov. 17-18, 2012 Manila (Philippines).

[7] DANG Yuan-yuan, FU Xiao-lin, XU Li-xin. Research on the Application of Agile Project Management with Scrum [J]. JOURNAL OF INTELLIGENCE, Mar. 2009.

[8] Lin Xie, Wei Pan. Personnel management of Scrum agile software development, 2010 International Conference on Future Information Technology and Management Engineering.

[9] CHEN Guo-dong, LUO Sheng-xian, Improvement and Application of Scrum Agile Software Development Method in Practice, Dec. 2011, Computer Technology and Development.

[10] Modeling and Simulation of Agile Manufacturing Cell. Modeling and Simulation of Agile Manufacturing Cell [J]. Proceedings of ICMEM2007 International Conference on Mechanical Engineering \& Mechanism November 5-7, 2007, Wuxi, P.R.China.

[11] Yuan Xiao, Hao Dongjing, Liu Haiyan, Jin Zhefeng, Dong Dayong. Civil aircraft cockpit evaluation of aircraft design and research method [J]. Civil Aircraft Design and Research, 2017 (1): 17-22.

[12] China Civil Aviation Administration 2nd China civil aviation regulations twenty-fifth: Transport Aircraft Airworthiness Standard [S]. China Civil Aviation Administration, 2001. 
[13] Jinru Teng, Yawei Song, Tao Wang. The Development of the Rugby Scrum Training Equipment [J], Applied Mechanics and Materials Vol. 440 (2014) pp 329-332.

[14] N. Zabkar, T. Hovelja, J. Urevc, V. Mahnic. Introducing Agile Software Development: Lessons Learned from the First Scrum Project in a Slovenian Company [S]. 2015 Intl. Conference on Advances in Management Engineering and Information Technology (AMEIT 2015). 